\title{
Coherent Fibre Bundles in full-field swept-source OCT
}

\author{
Helen D Ford ${ }^{*}$ and Ralph P Tatam \\ Engineering Photonics Group, School of Engineering, Cranfield University, Cranfield, Bedford, \\ MK43 0AL, UK
}

\begin{abstract}
Endoscopic OCT probes deliver light to the measurement region via a single optical fibre mounted in a probe head. The output beam is focused onto the sample, providing a single point measurement. The beam is translated, using mechanical scanning at the probe tip, to address a line or area of sampling points and produce an image.

We are investigating a swept-source OCT system incorporating coherent fibre bundles, to allow many measurement points to be addressed, within an area of the sample, without the need for mechanical motion within the endoscope probe. Scanning components are still present at the input of our system, but are no longer required within the flexible endoscope section. This allows a small-diameter, electrically passive probe to be engineered using off-the-shelf scanning components. A common-path probe design is proposed, in which the bundle is external to the OCT interferometer. This eliminates contrast variations caused by non-controllable differences in the state of polarisation between fibres. Imaging bundle fibres are typically few-moded, which can lead to ghost features and reduced SNR in OCT images, but the common-path configuration also removes cross-mode interference problems, and reduces dispersion artefacts.

OCT images of a microscope cover-slip and a sample of spring onion, acquired using the swept-source, bundle-based OCT system are presented. Features peculiar to the inclusion of the fibre bundle are discussed, and directions for future development of the system are outlined.
\end{abstract}

Keywords: OCT, optical coherence tomography, coherent fibre bundles, imaging bundles, swept-source

\section{INTRODUCTION}

Current endoscopic OCT probes comprise a single fibre mounted in an external housing ${ }^{1,2}$. Lenses are used to focus the output beam from the delivery fibre onto the sample of interest, providing an OCT measurement at a single point. For tomographic imaging, the beam is scanned in one or two dimensions to address a line or area of sampling points within the region of interest. A variety of methods have been investigated to achieve this; the use of rotating components within the endoscope tip provides imaging over an arc centred on the probe, which is particularly suitable for looking at the internal surfaces of cylindrical samples ${ }^{3}$. Implementations involving miniature scanners or MEMS devices have also been demonstrated, which commonly address a rectangular region of the sample surface ${ }^{4}$.

While successful, such miniaturised scanning systems can be awkward to implement in a small-diameter probe, and require electrical power to be delivered to the endoscope tip. We have previously investigated the use of a coherent fibre imaging bundle, to allow spatial multiplexing of OCT measurements from many sampling points without the need for scanning within the endoscope tip ${ }^{5}$.

A coherent fibre bundle, also sometimes known as an imaging fibre bundle, is an ordered arrangement of very smalldiameter optical fibres ${ }^{6}$. Typically, the bundle is comprised of many thousand fibres, each of which occupies the same relative position in the matrix at either end of the bundle. Thus an image projected onto one end of the bundle is transmitted to the opposite end without distortions, other than any variations between the optical loss for individual fibres. There is also a pixelation effect resulting from loss of light in the non-guiding regions between the fibre cores, which results in a fill-factor of less than one.

Our previous work on fibre-bundle OCT systems was based on a time-domain (TD) OCT system, using a broadband source and mechanical path-length scanning ${ }^{5}$. The entire input face of the bundle was illuminated, and measurements from all fibres acquired simultaneously using a CCD camera. Imaging was achieved using the bundle-based TD-OCT system, but the use of a camera imposed certain limitations. The frame rate was relatively slow and, because the source

\footnotetext{
*h.d.ford@cranfield.ac.uk; Telephone +44 (0)1234 750111; cranfield.ac.uk/soe/departments/processsystems/engineeringphotonics
} 
light was divided between many hundreds of fibres, light levels were low at individual imaging points, necessitating long integration times. Phase drift of the OCT fringes between frames was therefore a problem, and the pre-digitisation filtering techniques which permit DC removal and envelope detection in point OCT systems were not available, limiting imaging depth and contrast.

Some previous work by a group from California discusses an alternative method for the use of bundles in a time-domain OCT system ${ }^{7}$. In this work, the bundle was placed in the sample arm of a fibre Michelson OCT interferometer. The focusing optics were mounted on a translation stage to allow beam-scanning across the input end of the bundle. Imaging of a rabbit trachea was achieved successfully with this system.

In our current work on bundles for OCT, we also employ beam scanning at the input to the bundle. However, our system uses swept-source illumination. This has many advantages, both in terms of the optical design possibilities and the potential image contrast that is achieved when frequency-domain processing is applied.

Swept-source processing removes the requirement for a depth-scanning component, which allows us to implement a common-path probe design in which the bundle is used as a light-conduit, but does not form part of the OCT interferometer. This is advantageous for several reasons. Firstly, it is not at present possible to control the state of polarisation (SOP) within all (or indeed any) of the individual fibres of a coherent bundle. Investigation of the output SOP from a bundle, when light of a common SOP illuminates the input face, is found to vary significantly between fibres ${ }^{8}$. Thus fringe visibility is likely to be very variable between fibres, leading to poor contrast in some parts of the image when the bundle resides within the interferometer. Secondly, an imaging bundle has a high NA, and the fibres are typically few-moded at wavelengths within the transmission spectrum. This can lead to ghost images, as reported by Xie et $a l^{7}$, if multiple spatial modes participate in the interference process. By removing the bundle from the interferometer, the possibility of ghosting is eliminated. The common path arrangement also eliminates problems due to different dispersion in the two interferometer arms.

Coherent fibre bundles typically contain 10,000-100,000 fibres with core diameters ranging from a few micrometres to a few tens of micrometres. For a probe with unit magnification, the lateral resolution of the instrument is equal to the core spacing. The depth resolution is determined by the sweep range of the laser source. For the $1300 \mathrm{~nm}$ source used in this study, which has a sweep range of about $100 \mathrm{~nm}$, a depth resolution of about $20 \mu \mathrm{m}$ in air would be expected.

\section{TRANSMISSION PROPERTIES OF COHERENT FIBRE BUNDLES}

Standard coherent bundles are intended primarily for use in the visible. Those that we have used (sold by Schott) are typically few-moded in the middle of the visible range. Internal losses in bundles are higher than in single fibres due to a combination of factors: firstly, for flood-illumination of the bundle, there is the loss of light that impinges on regions outside the fibre cores, and is therefore unguided. There is also some absorption in the fibres, and coupling losses can be important because the NA of bundles is much higher than that of individual fibres, at around 0.5-0.6. The overall loss for Schott bundles is quoted at around $40 \%$ for a 1 metre bundle in the visible region of the spectrum. The quoted loss typically includes reflections of about $4 \%$ at both the proximal and distal polished surfaces.

Reflective losses, clearly, occur also for single optical fibres though, for both single fibres and bundles, they can be suppressed by angle-polishing the ends of the guide and/or application of coatings to the end surfaces. The quoted bundle loss must be treated with caution, since fill-factor losses are not applicable if light is focused into an individual core within the bundle, as is sometimes the case in this work. Specialist bundles for the near IR are available and should perform better for these wavelengths than the standard type.

We have investigated the performance of two types of imaging bundle supplied by Schott. Both are made from types of crown glass. Wound bundles are formed by stacking and then drawing square-packed "sub-bundles" (comprising 5 x 5 fibres) down to a side dimension of $50 \mu \mathrm{m}$, winding these sub-bundles onto a mandrel, embedding the region designed to form the end-sections into a resin matrix, then cutting and polishing the ends. Leached bundles are manufactured from many thousand hexagonally-packed rods, comprising core, cladding and secondary cladding fused into a rigid bar, drawing the rod to achieve the required core diameter, then etching away the secondary cladding from all except the end sections to leave a flexible image conduit.

In the visible region of the spectrum, the transmission of both wound and leached bundles was as expected. However, for OCT, the optimum wavelengths for use with tissue are in the near infra-red. The bundle transmission was also measured, therefore, in this region. The transmission drops off at the higher wavelengths, and the glass exhibits an inconvenient $\mathrm{OH}$ absorption at around $1400 \mathrm{~nm}^{9}$ which, since the scanning laser operates in the range $1280-1380 \mathrm{~nm}$, affects the upper end of the sweep range particularly severely. The overall transmission of a $1350 \mathrm{~mm}$ long bundle, measured with the swept 
source, was only around 6-8\%, including fill-factor and end-reflection losses. Since the beam must traverse the bundle twice in the OCT system, less than $1 \%$ of the initial coupled power is returned to the detector, ignoring all other losses in the system. We will address this problem in two ways. The simplest means to minimise transmission losses is to use a shorter bundle; lengths of about $0.5 \mathrm{~m}$ are readily available. Another possibility is to use a bundle specifically designed for use in the infra-red. These are now available and should offer much-improved performance, though the spectral transmission characteristics for the entire wavelength range of interest are not readily available.

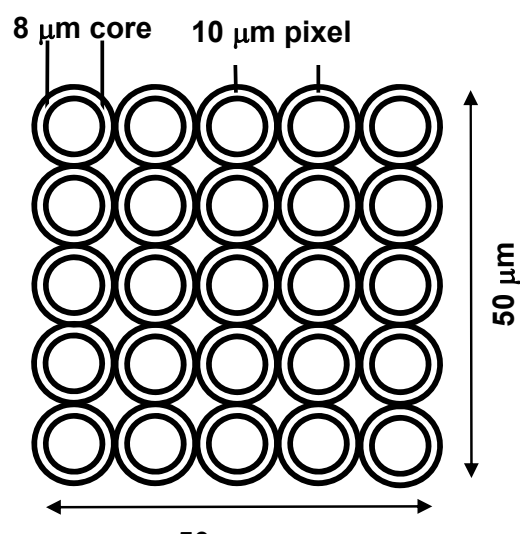

$50 \mu \mathrm{m}$
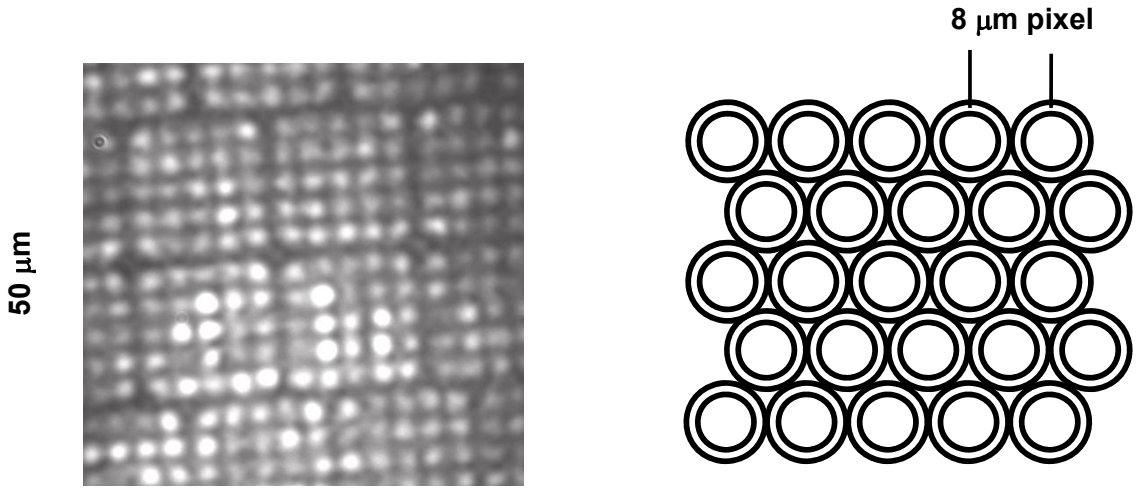

Figure 1. Schematic diagrams of Schott imaging bundles. Left, a sub-bundle from a wound bundle, with photograph of a larger region of the bundle, showing square packing with irregularities. Right, schematic of a leached bundle, showing hexagonal close packing of the fibres.

It is also important for the use of bundles in OCT that internal cross-coupling between the fibres should be negligible. As wavelength increased, the two types of bundle differed in their ability to confine the injected light to a particular fibre core within the bundle. In the leached bundle, $1300 \mathrm{~nm}$ light was well-confined to a single core but, at $1550 \mathrm{~nm}$, light focused into a single core emerged at the distal end from multiple cores. Despite the presence of interstitial absorbers between the fibre cores of the sub-bundles, the wound bundle was unable to confine light to a single core at the lower wavelength of $1300 \mathrm{~nm}$. Light coupled into a single fibre emerged from the entire group of 25 fibres in the local subbundle, although one fibre often tended to be more strongly illuminated than the others. At $1550 \mathrm{~nm}$, the cross-coupling became stronger, and the output light was more equally distributed between the fibres of the sub-bundle, even coupling partially to neighbouring sub-bundles. Thus we concluded that, although the leached bundle could be used in a $1300 \mathrm{~nm}$ OCT system, the type of wound bundle investigated was unsuited for use in OCT at NIR wavelengths.

\section{BUNDLE-BASED SWEPT-SOURCE OCT PROBE DESIGN}

\subsection{General description}

The OCT probe design, shown in figure 1, incorporates a coherent fibre bundle and a high-speed swept laser source at $1330 \mathrm{~nm}$ centre wavelength. The system operates in the spectral domain, with a fixed path-length imbalance.

Mechanical scanning of the beam is still required, but it is now positioned remotely, at the input to the bundle, while mechanical motion of components within the endoscopic section is avoided. A common-path configuration has been chosen. This is attractive when using bundles, because it avoids problems associated with polarisation variations between the bundle fibres; it is difficult to see how polarisation-matching could be achieved for all fibres of the bundle simultaneously in the popular Michelson interferometer configuration. The polarisation state in a proportion of the fibres will always be poorly matched to that of the reference beam, resulting in low contrast for these imaging positions.

A Fizeau arrangement is possible, with the reference beam derived either from the distal bundle end reflection or from an external reflector ${ }^{10}$. These are both of interest, particularly in benchtop applications, as they allow for very compact optics at the probe tip. However, both do have drawbacks. If the reference is derived from the fibre end reflection, a 
balancing interferometer will almost certainly be required to match the relatively long path length within the probe interferometer, and this reduces the signal-to-noise performance that can be achieved. An external reflector avoids the necessity for a balancing interferometer, but is more difficult to arrange practically, with an appropriate path-length imbalance, in a system designed for endoscopic use. Therefore, a third alternative, shown in the figure, was selected for this investigation.

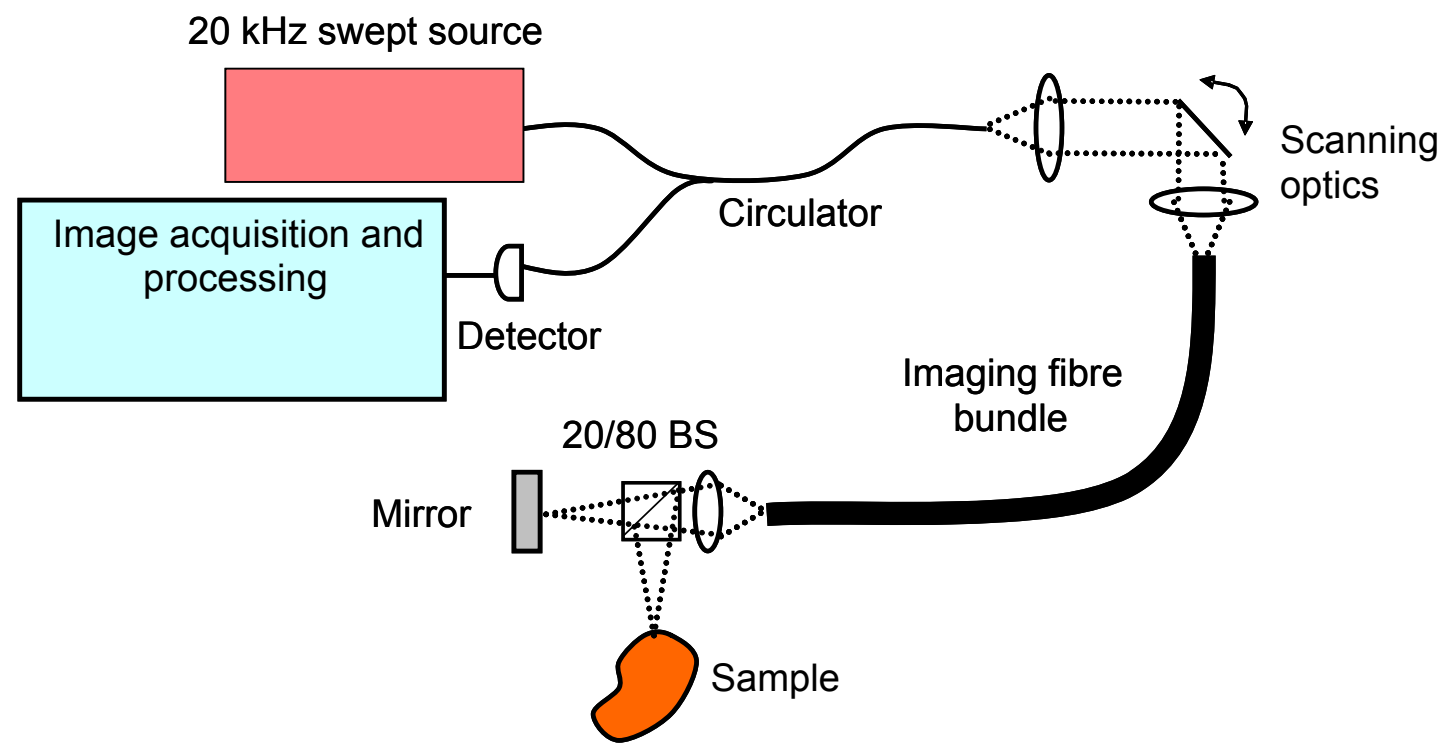

Figure 2. Schematic of a common-path OCT imaging system using a Michelson interferometer at the probe tip and incorporating a coherent bundle with a $20 \mathrm{kHz}$ swept wavelength source.

A miniature Michelson interferometer was constructed at the probe tip, using a custom 20/80 beamsplitter cube, specially designed to perform well over a $100 \mathrm{~nm}$ bandwidth. The purpose of the unequal split ratio was to achieve a ratio of signal/reference beam powers appropriate for good visibility OCT fringes, without the necessity for attenuation of the reference beam. The 20/80 split ratio implies side-illumination of the sample, which is preferable in any case as it allows all the optical elements at the probe tip to be centred on the optical axis, allowing a relatively small probe diameter to be achieved by the use of small-diameter components. As with any design using an external reflector, suppression of the reflection from the distal end of the bundle is then necessary. This can be achieved by a combination of angle polishing the output surface (at an angle of $8-12^{\circ}$ ) and/or applying an anti-reflective coating to this surface.

\subsection{Scanning optics}

The output beam from the swept source is delivered via a single-mode optical fibre. Light is routed from the laser, via a Thorlabs INT-COM optoelectronic module containing a broadband circulator, to the optical bench. The module also contains a fibre wavelength multiplexer, to allow a visible red laser to be mixed with the $1300 \mathrm{~nm}$ light for aiming purposes.

All fibre connections between the laser output and the OCT probe input have angle-polished end connectors to minimise stray reflections. At the output fibre, an aspheric lens is used to collimate the beam onto the scanner. At present this is a galvanometer mirror with a single scan axis. This will shortly be upgraded to allow for either 2D scanning in an arbitrary direction, or full 3D acquisition. A second aspheric lens focuses the scanned beam onto the bundle input face such that the beam waist is smaller than the core diameter of a single fibre. Thus individual fibre cores can be addressed in turn. 


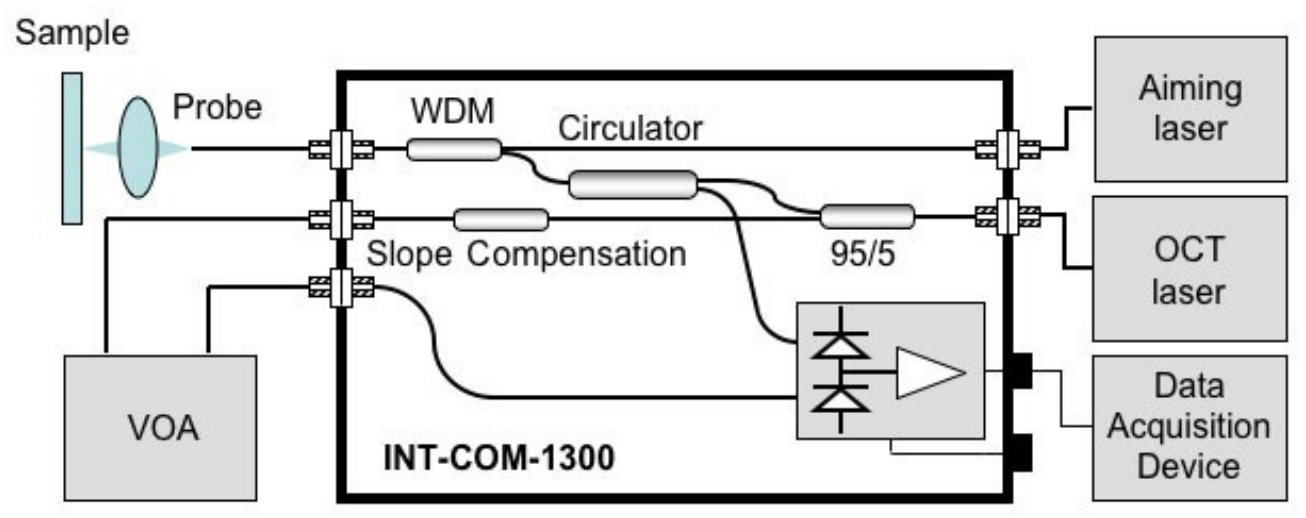

Figure 3. Schematic of Thorlabs INT-COM light delivery and balanced detector module for common path OCT systems.

The beam is currently scanned raster-fashion across the input end of the bundle. The scan rate for the beam is selected to ensure a quasi-static beam during the $50 \mu$ duration of the wavelength sweep. Step-and-settle scanning, where the beam is moved rapidly between fibres, stopping at each fibre in turn, will also be investigated. However, this is likely to prove slower, given the scan accuracy required to achieve optimum coupling into each fibre in the bundle. The mirror scan is synchronised with the acquisition of data; a fast digitiser collects many hundred data points in the $\mathrm{z}$ dimension (depth) for each laser sweep. When this is repeated for each of the several hundred fibres within one raster, 2D data can be acquired and displayed very rapidly. A raster scan rate of 80 Hertz has been achieved using this method. Once scanning is extended to the other dimension, full-field data of about $200 \times 200$ pixels is expected to be acquired in $1-5$ seconds.

\subsection{Probe optics}

The leached imaging bundle has an NA in excess of 0.5 ; much higher than the typical NA of about 0.1 that characterises a standard single mode optical fibre. A large NA is an advantage at the input end, as light can be injected into the bundle with a low coupling loss. Although the large NA can lead to multi-moding, this is generally avoided by using an input lens with a much lower NA. However, the situation in the probe tip is more complicated. A large NA results in a small beam waist, but a poor Rayleigh range, so it becomes necessary to reduce the NA to improve the depth of focus. This in turn leads to a magnification of greater than 1 and reduced lateral resolution, so a compromise must be reached between all the interdependent imaging parameters.

It is not desirable to work with a very large magnification. Although a larger sample region can be imaged with each raster the lateral resolution, which is limited by the bundle-fibre core separation, also increases, and small details of the sample structure will be obscured. It was decided, in our system, to work with a magnification of 2.5-3. This provides a Rayleigh range of about $100 \mu \mathrm{m}$ with a lateral resolution of 25-30 $\mu \mathrm{m}$.

In contrast to a single-fibre OCT probe, the focusing optics must ensure low-loss, low-aberration imaging for off-axis object points. The field of view for the probe lens is typically rather small, since the coherent bundle diameter is unlikely to exceed $2 \mathrm{~mm}$. However, given the other constraints on the system, significant care is nevertheless required to ensure adequate operation towards the periphery of the images. The use of high-quality lenses is essential. At present, with a 1 $\mathrm{mm}$ diameter bundle, aspheric lenses result in well-focused images of peripheral fibres but, for larger diameter bundles, coated, multi-element lenses are likely to be the best solution.

\subsection{Data acquisition and processing}

Light returning from the OCT probe re-enters the delivery fibre, and is directed by the circulator to one arm of a balanced detector contained within the Thorlabs INT-COM module. Despite the use of angled-polish FC/APC connectors in the delivery fibres, sufficient light is returned from the fibre connections to give an appreciable DC level at the detector. A small amount of light, picked off from the output fibre using a 95/5 coupler, and routed to the second arm of the balanced detector via a variable optical attenuator, allows much of this stray light to be balanced out, thereby improving OCT contrast. 
LabView is used for control and processing. The output from the detector is passed to a high-speed digitiser card, synchronised both to the $20 \mathrm{kHz}$ laser scanning frequency and to the scanning mirror, via a position sensor in the mirror drive circuit. The required number of sampling points per laser sweep, and the overall rate of sample acquisition, can be selected at will. For work carried out to date using this system, about 800 sample points have been acquired for each sweep of the laser. This is ample for sampling of fringes corresponding to an imaging depth of several millimetres. The sample number determines the required sampling rate, since the laser repeat frequency of $20 \mathrm{kHz}$ is fixed. The total number of A-scans per raster is currently set to about 200 . Since the number of fibres across the diameter of the bundle is of the order of 100, this ensures that each fibre on the raster is sampled at least once, assuming good coupling into the fibres at all times.

A Fourier transform is performed on the acquired data from the digitizer, and the logarithm of the result is calculated to produce the OCT images. The program provides a real-time display. Although the number of lateral samples is userselectable, the lateral resolution is ultimately determined by the fibre core separation, and the lateral image size by the diameter of fibre bundle used.

In practice, unless the orientation of the rows of bundle fibres is perfectly aligned with the raster scan, the coupling will vary periodically. This is illustrated in figure 4(a). If we assume a focused beam waist no larger than the core diameter, good coupling is achieved when the beam is reasonably well-centred on the fibre core. This is the case over a certain portion of the scan, and then coupling decreases gradually as the beam moves off towards an interstitial 'dead spot'. Coupling then begins to improve again, and the cycle repeats across the width of the bundle. Image smoothness should be improved, at the expense of a small reduction in lateral resolution and power at the detector, by enlarging the beam waist at the bundle input, to a diameter larger than that of the fibre cores, as shown in figure 4(b). At this end of the bundle, where compactness is less critical, a large-diameter lens is helpful to reduce losses for light returning to the detector.

(a)

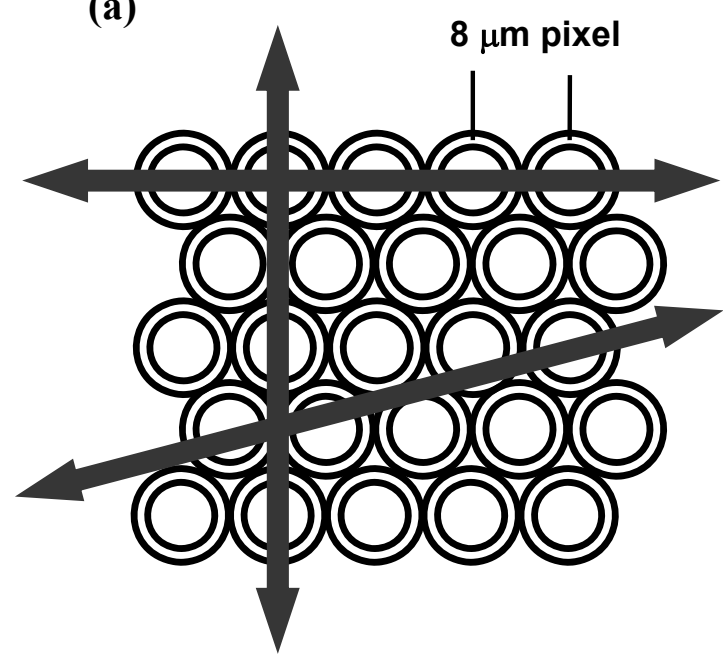

(b)

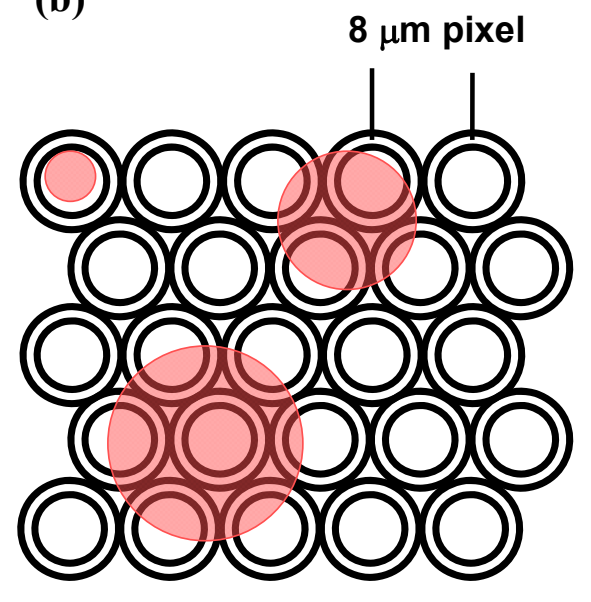

Figure 4. Illustrating how: (a) coupling to bundle fibres varies depending on the relative orientation of fibre alignment and raster direction. (b) beam waist relative to fibre diameter affects coupled intensity at each imaging position.

\section{RESULTS}

The system was tested, initially on highly-reflective glass and plastic samples and subsequently on samples of vegetable tissue. Results from a glass microscope cover slip are shown in figure 5(a). The front and rear surfaces, represented by the top and bottom lines in the image, are clearly visible, separated by about $200 \mu \mathrm{m}$. The intermediate line is due to a self-interference between the front and rear surfaces of the cover slip, and appears because the reflected intensities for this object are comparable to that of the reference reflection. Unlike the other two lines in the image, this line does not 
move relative to the image frame as the object is translated along the optical axis, because the path-length difference is fixed by the object thickness.

Figure 5(b) shows preliminary results from a sample of spring onion. The SNR is low in this image, probably as a result of the very high bundle transmission loss, but two layers can be clearly distinguished, separated by about $100 \mu \mathrm{m}$. Features arising from the bundle characteristics are visible within the images. There is a distinctive vertical 'striping', which corresponds to illumination by individual fibres, and a lack of signal in the non-transmitting interstitial regions. The striping is prominent here because the image represents quite a small number of image points at the system resolution; the number of fibres across the image is about 65 . The appearance could be smoothed by binning. However, it would also be desirable to select a bundle, perhaps of greater diameter, containing a larger number of fibres, of smaller core diameter and separation. This would give a greater density of data points within the image.

Another feature of figure 5(b) is the periodic variation in intensity across the image, attributed to the effect described above, where the coupling of input light to the bundle fibres varies within the raster scan. At present we do not have the ability to scan in two dimensions, but the same effect can be simulated by translating the bundle in a direction normal to the line of the raster. When the bundle is moved manually in this way through a few micrometres, the regions of maximum illumination move laterally, as expected. Figure 5(c) is an average of five OCT images, taken from a region of the sample close to that shown in figure 5(b). In this figure it is possible to distinguish three layers. The bundle has been translated normal to the raster by about $2 \mu \mathrm{m}$ between each of the five acquisitions, and the lateral intensity variation is now much less pronounced.

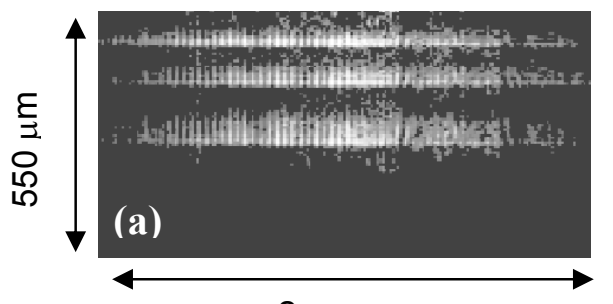

$2 \mathrm{~mm}$
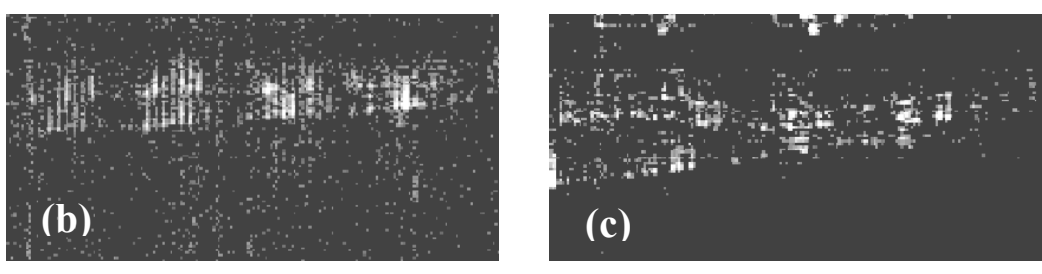

Figure 5. OCT results from above (a) a $200 \mu \mathrm{m}$ thick microscope cover slip, and below a sample of spring onion showing (b) single-shot image with two visible layers, (c) an average of five images, displaying three visible layers, with translation of the raster between acquisitions.

\section{DISCUSSION}

OCT imaging using a swept-source OCT probe incorporating a coherent fibre bundle has been demonstrated for both a highly reflective and a biological sample. The system resolution is currently about $30 \mu \mathrm{m}$ in the lateral direction and about $20 \mu \mathrm{m}$ in depth (both defined in air). The Rayleigh range is about $100 \mu \mathrm{m}$. The maximum number of fibres per raster, corresponding to a diameter of the imaging bundle, is about 100, which currently places the limitation of the density of imaging points for a single raster. This could be increased for the existing bundle by translating the sample and merging adjacent images.

Imaging bundles are also available from other manufacturers. These alternative bundles are fused throughout their length, which makes them much less flexible than the Schott bundles, and are manufactured from doped silica, rather than glass. The transmission properties in the near infra-red are not well documented. The core shape and spacing are both irregular, unlike the close-packed, circular cores of the Schott bundles, and the fill factor tends to be lower ${ }^{11}$. The irregularity of the cores serves to prevent inter-fibre coupling, which increases as cores become smaller and more closely spaced $^{12}$, and in such bundles the cores can be as small as $2 \mu \mathrm{m}$, which offers a potential increase in lateral resolution. These components therefore merit investigation for use in OCT.

Our immediate plans for development of our swept-source bundle-based OCT system are to implement two-dimensional scanning and to investigate the effect of substituting either a shorter, or an IR-specific bundle for the current $1350 \mathrm{~mm}$ long bundle. We expect thereby to improve image contrast significantly and expand the range of sample types accessible to our system. In the medium term we aim to improve the data density of our images by the acquisition of alternative bundles, containing more fibres of smaller core size and spacing. 
A loss of contrast occurs at present, towards the periphery of the images, for a full scan across the entire diameter of the bundle. This is because of beam 'walk-off' from the reference mirror for large-angle rays. Modification of the optical arrangement in the probe-end imaging system will reduce this problem.

\section{ACKNOWLEDGMENTS}

The authors acknowledge the award of a grant (EP/F034679/1) from the Engineering and Physical Science Research Council (EPSRC), UK.

\section{REFERENCES}

[1] Feldchtein, F. I., Gelikonov, V. M. and Gelikonov, G. V., [Handbook of Optical Coherence Tomography], Informa Health Care, London, New York, Melbourne and Stockholm, 125-143 (2002).

[2] Han, S., Sarunik, M. V., Wu, J., Humayun, M. And Yang, C., "Handheld forward-imaging needle endoscope for ophthalmic optical coherence tomography inspection", Journal of Biomedical Optics Letters, 13(2), no. 020505 (2008).

[3] Tearney, G. J., Brezinski, M. E., Bouma, B. E., Boppart, S. A., Pitris, C., Southern, J. F. and Fujimoto, J. G., "Invivo endoscopic optical biopsy with optical coherence tomography", Science, 276(5321), 2037-2039 (1997).

[4] Xu, Y., Singh, J., Premachandran, C. S., Khairyanto, A., Chen, K.W.S., Chen, N., Sheppard, C. J. R. and Olivo, M., "Design and development of a 3D scanning MEMS OCT probe using a novel SiOB package assembly", J. Micromech. Microeng. 18, no. 125005 (2008).

[5] Ford, H. D. and Tatam, R. P. "Full-field optical coherence tomography", Proc. SPIE 5858-19 (2005).

[6] http://www.schott.com/fiberoptics/english/products/healthcare/imagingfiberoptics/flexiblecomponents

[7] Xie, T., Mukai, D., Guo, S., Brenner, M. and Chen, Z., "Fibre-optic-bundle-based optical coherence tomography", Opt. Lett. 30(14), 1803-1805, (2005).

[8] Sarantavgas, G., Ford, H. D. and Tatam, R. P., "Imaging fibre bundles for Fizeau based optical coherence tomography", Proc. SPIE 6847-0C, (2008).

[9] Gusarov, A., Doyle, D., Glebov, L. and Berghmans, F., "Comparison of radiation-induced transmission degradation of borosilicate crown optical glass from four different manufacturers", Proc. SPIE 5897-01 (2005).

[10] Casaubieilh, P., Ford, H. D., James, S. W. and Tatam, R. P., "Optical coherence tomography with a Fizeau interferometer configuration", Proc. SPIE 5858-18, (2005).

[11] Udovich, J. A., Kirkpatrick, N. D., Kano, A., Tanbakuchi, A., Utzinger, U. And Gmitro, A. F., "Spectral background and transmission characteristics of fiber optic imaging bundles", Appl. Opt., 47(25), 4560-4568 (2008).

[12] Reichenbach K. L. and Xu, C., "Numerical analysis of light propagation in image fibers or coherent fiber bundles", Opt. Express, 15(5), 2151-2165 (2007). 